This item was submitted to Loughborough's Research Repository by the author.

Items in Figshare are protected by copyright, with all rights reserved, unless otherwise indicated.

\title{
Reachability problems for hierarchical piecewise constant derivative systems
}

PLEASE CITE THE PUBLISHED VERSION

http://dx.doi.org/10.1007/978-3-642-41036-9_6

PUBLISHER

(C) Springer-Verlag Berlin Heidelberg

VERSION

AM (Accepted Manuscript)

LICENCE

CC BY-NC-ND 4.0

REPOSITORY RECORD

Bell, Paul C., and Shang Chen. 2019. "Reachability Problems for Hierarchical Piecewise Constant Derivative Systems". figshare. https://hdl.handle.net/2134/13390. 


\title{
Reachability Problems for Hierarchical Piecewise Constant Derivative Systems
}

\author{
Paul C. Bell and Shang Chen \\ Department of Computer Science, Loughborough University. \\ P.Bell@lboro.ac.uk, S.Chen3@lboro.ac.uk
}

\begin{abstract}
In this paper, we investigate the computability and complexity of reachability problems for two-dimensional hierarchical piecewise constant derivative (HPCD) systems. The main interest in HPCDs stems from the fact that their reachability problem is on the border between decidability and undecidability, since it is equivalent to that of reachability for one-dimensional piecewise affine maps (PAMs) which is a long standing open problem. Understanding the most expressive hybrid system models that retain decidability for reachability has generated a great deal of interest over the past few years. In this paper, we show a restriction of HPCDs (called RHPCDs) which leads to the reachability problem becoming decidable. We then study which additional powers we must add to the RHPCD model to render it 1D PAM-equivalent. Finally, we show NP-hardness of reachability for nondeterministic RHPCDs.
\end{abstract}

\section{Introduction}

Hybrid automata are an important class of mathematical model allowing one to capture both discrete and continuous dynamics in the same framework. There is currently much interest in hybrid systems since they can be used to model many practical real world systems in which we have a discrete controller acting in a continuous environment and their analysis has a huge range of potential applications, such as aircraft traffic management systems, aircraft autopilots, automotive engine control [6], chemical plants [7] and automated traffic systems for example.

Hybrid systems are described by a state-space model given by the Cartesian product of a discrete and continuous set. The system evolves over time according to a set of defined rules until some condition or event is satisfied, at which point a discrete, non-continuous event occurs. Such an event can cause an update to certain variables and change the continuous dynamics of the continuous variables.

A fundamental question concerning hybrid systems is that of reachability: does there exist a trajectory starting from some initial state (or set of states) which evolves to reach a given final state (or set of states) in finite time? Related questions, such as convergence (does there exist a state (or periodic set of states) towards which the system converges for any initial state) or control problems (given an input, can the system be controlled to avoid some 'bad' set of 
states?), are also important, see [9]. In this paper we focus on reachability. Unfortunately, many reachability problems are undecidable, even for very restricted hybrid systems $[2,5,8,10]$. The objective of studying the decidability boundary is twofold; to obtain the most expressive system for which reachability is decidable and to study the simplest system for which it is undecidable.

An important and intuitive model of hybrid system is that of a Piecewise Constant Derivative (PCD) system. In this model, we partition the continuous state space into a finite number of nonempty regions, each of which is assigned a constant derivative defining the dynamics of a point within that region (see Section 2 for full details). It was proven in [12] that reachability for PCD systems in two-dimensions (2-PCD) is decidable, but for three-dimensions (a 3-PCD), the problem becomes undecidable [2]. One of the important properties of a PCD, which leads to its reachability problem being decidable in dimension 2 , is that trajectories can never 'cross' each other since each region has a constant derivative assigned. It can be proven that the trajectories are either periodic, or else form an expanding or contracting spiral which can be proven using geometric arguments on the edge-to-edge successor function of a 2-PCD.

In [4], an intermediate model, called a Hierarchical Piecewise Constant Derivative (HPCD) system was introduced. Intuitively, this model of linear hybrid system can be thought of as a two-dimensional hybrid automaton where the dynamics in each discrete location is given by a 2-PCD (precise details are given in Section 2). Certain edges in locations of the HPCD are denoted as guards (which can be comparative) and lead to discrete location changes. When changing location, an affine reset rule may also be applied to the continuous variables. If all regions of the underlying PCDs are bounded, then the HPCD is called bounded. Clearly then, the model of HPCD seems more powerful than that of a 2-PCD. Indeed, the reachability problem for a one-dimensional Piecewise Affine Map (1-PAM) was shown to be equivalent to that of reachability for a bounded HPCD with either: i) comparative guards, identity resets and elementary flows in Proposition 3.20 of [3] or else ii) affine resets, non-comparative guards and elementary flows in Lemma 3.4 of [3] (See Section 2 for definitions).

Our reference model in this paper is called a Restricted HPCD (an RHPCD). An RHPCD is an HPCD with elementary flows, identity resets and non-comparative guards and is thus a simpler form of HPCD. We prove that reachability for an RHPCD is decidable. We also prove that a 1-PAM can also be simulated by an RHPCD with arbitrary constant flows or with linear resets, and is thus equivalent to an RHPCD with affine resets, see Table 1 for an overview of results.

In [13], the reachability problem for planar linear hybrid automata without resets is shown to be decidable, however they focus on the setting in which the flows are monotonic, meaning there exists some vector $\rho$ such that the derivatives of all variables in all states have a positive projection along $\rho$. In dimension 4 , the reachability problem becomes undecidable [13]. 


\begin{tabular}{|c|c|c|c|c|c|c|}
\hline \multirow{2}{*}{ RHPCD } & $\begin{array}{c}\text { Infinite number } \\
\text { of PCD regions }\end{array}$ & $\begin{array}{c}\text { Linear } \\
\text { resets }\end{array}$ & $\begin{array}{c}\text { Affine } \\
\text { resets }\end{array}$ & $\begin{array}{c}\text { Comparative } \\
\text { guards }\end{array}$ & $\begin{array}{c}\text { Arbitrary } \\
\text { constant flows }\end{array}$ & $\begin{array}{c}\text { Number of } \\
\text { locations }\end{array}$ \\
\hline \multirow{2}{*}{ Decidable } & $\times$ & $\times$ & $\times$ & $\times$ & $\times$ & $N<\infty$ \\
\cline { 2 - 7 } & $\times$ & $\times$ & $\times$ & $\checkmark$ & $\checkmark$ & 1 \\
\hline \multirow{2}{*}{$\begin{array}{c}\text { 1-PAM } \\
\text { equivalent }\end{array}$} & $\times$ & $\times$ & $\times$ & $\times$ & $\checkmark$ & $\left\lceil\log _{2} n\right\rceil+3$ \\
\cline { 2 - 7 } & $\times$ & $\times$ & $\times$ & $\checkmark$ & $\times$ & $4 n$ \\
\cline { 2 - 7 } & $\times$ & $\times$ & $\checkmark$ & $\times$ & $\times$ & 1 \\
\hline Undecidable & $\checkmark$ & $\times$ & $\times$ & $\times$ & $\times$ & {$[3]$} \\
\hline
\end{tabular}

Table 1. RHPCD (starred results are contributions of this paper)

\section{Preliminaries}

Intervals of the form $(s, t),[s, t),(s, t],[s, t]$ are called open, half-open or closed bounded rational intervals (respectively), where $s, t \in \mathbb{Q}$. We write $\langle I, c\rangle$ to denote $\{(x, c) \mid x \in I\} \subseteq \mathbb{Q}^{2}$, where $I \subseteq \mathbb{Q}$ is an (open, half-open or closed) bounded rational interval and $c \in \mathbb{Q}$ is a constant. We similarly define $\langle c, I\rangle=$ $\{(c, y) \mid y \in I\}$. By abuse of notation, for an interval $I=(s, t)$ where $s, t \in \mathbb{Q}$ and $s \leq t$, a function $f(x): \mathbb{Q} \rightarrow \mathbb{Q}$ and a constant $m \in \mathbb{Q}$, we define $f(I)+m=$ $(f(s)+m, f(t)+m)$. Similar definitions exist for half-open and closed intervals. We use similar definitions as [3] for the following.

Definition 1 (HA) An n-dimensional Hybrid Automaton (HA) [1] is a tuple $\mathcal{H}=\left(\mathcal{X}, Q, f, l_{0}\right.$, Inv,$\left.\delta\right)$ consisting of the following components:

(1) A continuous state space $\mathcal{X} \subseteq \mathbb{R}^{n}$. Each $\boldsymbol{x} \in \mathcal{X}$ can be written $\boldsymbol{x}=\left(x_{1}, \ldots, x_{n}\right)$, and we use variables $x_{1}, \ldots, x_{n}$ to denote components of the state vector.

(2) A finite set of discrete locations $Q$.

(3) A function $f: Q \rightarrow\left(\mathcal{X} \rightarrow \mathbb{R}^{n}\right)$, which assigns a continuous vector field on $\mathcal{X}$ to each location. In location $l \in Q$, the evolution of the continuous variables is governed by the differential equation $\dot{\boldsymbol{x}}=f_{l}(\boldsymbol{x})$. The differential equation is called the dynamics of location $l$.

(4) An initial condition $I_{0}: Q \rightarrow 2^{\mathcal{X}}$ assigning initial values to variables in each location.

(5) An invariant Inv: $Q \rightarrow 2^{\mathcal{X}}$. For each $l \in Q$, the continuous variables must satisfy the condition Inv(l) in order to remain in location $l$, otherwise it must make a discrete transition.

(6) A set of transitions $\delta$. Every $\operatorname{tr} \in \delta$ is of the form $\operatorname{tr}=\left(l, g, \gamma, l^{\prime}\right)$, where $l, l^{\prime} \in Q, g \subset \mathcal{X}$ is called the guard, defining when the discrete transition can occur, $\gamma \subset \mathcal{X} \times \mathcal{X}$ is called the reset relation applied after the transition from l to $l^{\prime}$.

An HA is deterministic if it has exactly one solution for its differential equation in each location and the guards for the outgoing edges of locations are mutually exclusive. A trajectory of a hybrid automaton $\mathcal{H}$ starting from $\left(l_{0}, \mathbf{x}_{\mathbf{0}}\right)$ where $l_{0} \in Q, \mathbf{x}_{\mathbf{0}} \in \mathcal{X}$ is a pair of functions $\pi_{l_{0}, \mathbf{x}_{\mathbf{0}}}=\left(\lambda_{l_{0}, \mathbf{x}_{\mathbf{0}}}(t), \xi_{l_{0}, \mathbf{x}_{\mathbf{0}}}(t)\right)$ such that 
(1) $\lambda_{l_{0}, \mathbf{x}_{0}}(t):[0,+\infty) \rightarrow Q$ is a piecewise function constant on every interval $\left[t_{i}, t_{i+1}\right)$.

(2) $\xi_{l_{0}, \mathbf{x}_{0}}(t):[0,+\infty) \rightarrow \mathbb{R}^{n}$ is a piecewise differentiable function and in each piece $\xi_{l_{0}, \mathbf{x}_{0}}$ is càdlàg (right continuous with left limits everywhere).

(3) On any interval $\left[t_{i}, t_{i+1}\right)$ where $\lambda_{l_{0}, \mathbf{x}_{\mathbf{0}}}$ is constant and $\xi_{l_{0}, \mathbf{x}_{\mathbf{0}}}$ is continuous,

$$
\xi_{l_{0}, \mathbf{x}_{\mathbf{0}}}(t)=\xi_{l_{0}, \mathbf{x}_{\mathbf{0}}}\left(t_{i}\right)+\int_{t_{i}}^{t} f_{\lambda_{l_{0}, \mathbf{x}_{\mathbf{0}}}\left(t_{i}\right)}\left(\xi_{l_{0}, \mathbf{x}_{\mathbf{0}}}(\tau)\right) d \tau
$$

for all $\tau \in\left[t_{i}, t_{i+1}\right)$.

(4) For any $t_{i}$, there exists a transition $\left(l, g, \gamma, l^{\prime}\right) \in \delta$ such that

(i) $\lambda_{l_{0}, \mathbf{x}_{\mathbf{0}}}\left(t_{i}\right)=l$ and $\lambda_{l_{0}, \mathbf{x}_{\mathbf{0}}}\left(t_{i+1}\right)=l^{\prime}$;

(ii) $\xi_{l_{0}, \mathbf{x}_{\mathbf{0}}}^{-}\left(t_{i+1}\right) \in g\left(l, l^{\prime}\right)$ where $\xi_{l_{0}, \mathbf{x}_{\mathbf{0}}}^{-}(t)$ means the left limit of $\xi_{l_{0}, \mathbf{x}_{\mathbf{0}}}$ at $t$;

(iii) $\left(\xi_{l_{0}, \mathbf{x}_{\mathbf{0}}}^{-}\left(t_{i+1}\right), \xi_{l_{0}, \mathbf{x}_{\mathbf{0}}}\left(t_{i+1}\right)\right) \in \gamma$.

Definition 2 (n-PCD) An n-dimensional Piecewise Constant Derivative ( $n$ PCD) system [2] is a pair $\mathcal{H}=(\mathbb{P}, \mathbb{F})$ such that:

(1) $\mathbb{P}=\left\{P_{s}\right\}_{1 \leq s \leq k}$ is a finite family in $\mathbb{R}^{n}$, where $P_{s} \subseteq \mathbb{R}^{n}$ are non-overlapping convex polygonal sets.

(2) $\mathbb{F}=\left\{\boldsymbol{c}_{s}\right\}_{1 \leq s \leq k}$ is a family of vectors in $\mathbb{R}$.

(3) The dynamics are given by $\dot{\boldsymbol{x}}=\boldsymbol{c}_{s}$ for $\boldsymbol{x} \in P_{s}$.

An n-PCD is called bounded if for its regions $\mathbb{P}=\left\{P_{s}\right\}_{1 \leq s \leq k}$, there exists $r \in \mathbb{Q}^{+}$, such that for all $P_{s}$, we have that $P_{s} \subseteq B_{\mathbf{0}}(r)$, where $B_{\mathbf{0}}(r)$ is an origin-centered open ball of radius $r$ and appropriate dimension.

We define the support set of a PCD $\mathcal{H}$ as $\operatorname{Supp}_{\mathrm{PCD}}(\mathcal{H})=\bigcup_{1 \leq s \leq k} P_{s}$. Given an edge $e$, we represent a point on $e$ by a one-dimensional local coordinate, allowing us to define an edge-to-edge successor function as an affine function between edges.

Definition 3 (HPCD) A Hierarchical Piecewise Constant Derivative (HPCD) system [3] is a hybrid automaton $\mathcal{H}=\left(\mathcal{X}, Q, f, l_{0}\right.$, Inv, $\left.\delta\right)$ such that $Q$ and $l_{0}$ are defined as in Definition 1, with the dynamics at each $l \in Q$ given by a 2$P C D$ and each transition $t r=\left(l, g, \gamma, l^{\prime}\right)$ is such that: (1) Its guard $g$ is a line segment in $\mathbb{R}^{2}$; and (2) The reset relation $\gamma$ is an affine function of the form: $\boldsymbol{x}^{\prime}=\gamma(\boldsymbol{x})=\boldsymbol{A} \boldsymbol{x}+\boldsymbol{b}$. We denote the internal guards of an HPCD location to be the guards of the underlying $P C D$ regions and the transition guards to be the guards used in transitions between locations. The Invariant (Inv) for a location $l$ is defined to be $\operatorname{Supp}_{P C D}(\mathcal{H}) \backslash \mathcal{G}_{l}$, where $\operatorname{Supp}_{P C D}(\mathcal{H})$ is the support set of the underlying PCDs of the HPCD and $\mathcal{G}_{l}$ is the set of transition guards in location l. If all the PCDs are bounded, then $\mathcal{H}_{P C D}$ is said to be bounded.

It was shown in [3] that reachability for an HPCD is equivalent to reachability for a 1-PAM. An HPCD system has elementary flows if the derivatives of all variables in each location are in $\{0, \pm 1\}$, otherwise it has arbitrary constant flows. Guards are defined as line segments, described by boolean combinations of linear 
inequalities. If each atomic formula contains only one variable $(x$ or $y)$, then the guard is called non-comparative. An HPCD has non-comparative guards if all guards are non-comparative, e.g., $\frac{3}{2} \leq x \leq 7 \wedge y=-1$ is non-comparative, but $0 \leq x \leq 1 \wedge 0 \leq y \leq \frac{1}{2} \wedge x=2 y$ is a comparative guard. We define an affine reset of variables $\mathbf{z}=(x, y)$ as $\gamma(\mathbf{z})=A \mathbf{z}+\mathbf{b}$ where $A \in \mathbb{Q}^{2 \times 2}$ and $\mathbf{b} \in \mathbb{Q}^{2}$, a linear reset is of the form $\gamma(\mathbf{z})=A \mathbf{z}$ and an identity reset is $\gamma(\mathbf{z})=\mathbf{z}$. Our reference model is called an RHPCD, a restricted HPCD. We later show that adding any one of the additional powers - arbitrary constant flows, comparative guards or linear resets - allows simulation of a 1-PAM. Note that since reachability for HPCDs is equivalent to reachability for 1-PAMs and an RHPCD with these powers is a restricted form of HPCD, then showing reachability for a 1-PAM can be simulated by such an RHPCD shows their equivalence. Equivalence of reachability between 1-PAMs and planar pseudo-billiard systems was shown in [11], whereas more complex 1-dim. functions allow universal computation.

Definition 4 (RHPCD) A Restricted Hierarchical Constant Derivative System (RHPCD) is a bounded HPCD with identity resets, non-comparative guards, elementary flows and a finite number of PCD regions. See Fig. 36 and Fig. 4 for an example of an RHPCD with arbitrary constant flows.

Our final model is the class of one-dimensional Piecewise Affine Maps (1PAM). The reachability problem for 1-PAM is currently a long-standing open problem, even for two intervals. Our approach follows a similar style to [3] where we show various classes where reachability is equivalent to that of a 1-PAM.

Definition 5 (1-PAM) - A one-dimensional Piecewise Affine Map (1-PAM) is a function $f: \mathbb{R} \rightarrow \mathbb{R}$ (See Fig. 3a for an example) such that:

(1) Domain of $f: \operatorname{dom}(f)=\bigcup I_{i}$, where $I_{i}$ are disjoint rational intervals.

(2) $\exists a_{i}, b_{i} \in \mathbb{Q}$ such that $\forall x \in I_{i}, f(x)=a_{i} x+b_{i}$.

(3) $f$ is closed, i.e., range $(f) \subseteq \operatorname{dom}(f)$.

Simulation : We use the definition of simulation of PCDs described in [2]. If model $\mathcal{A}$ can be simulated by model $\mathcal{B}$, then reachability for $\mathcal{A}$ can be reduced to reachability for $\mathcal{B}$ ( $\mathcal{A}$ has a decidable reachability problem as long as $\mathcal{B}$ does).

Reachability Problems : By an instance of a reachability problem we mean a finite description of a model, an initial and final configuration (or set of configurations). Reachability: starting from the initial configuration(s), does the trajectory eventually reach the final configuration(s) in finite time after a finite number of (discrete) transitions? Models of hybrid automata with an infinite number of transitions in finite time are said to have the Zeno property which we do not consider here. Problems such as convergence, stability and control are not considered in this paper, see [9] for more information about these problems.

Open Problem 6 - 1-PAM Reachability - Given a 1-dim. Piecewise Affine Map $f$, and points $x, y \in \mathbb{Q}$. Does there exist $t \in \mathbb{N}$, such that $f^{t}(x)=y$ ? ${ }^{1}$

\footnotetext{
$\overline{{ }^{1} f^{t}(x) \text { denotes }} \underbrace{f(f(\ldots f(x) \ldots))}_{t}$
} 


\section{Reachability for RHPCDs and Extensions}

We now explore reachability for our models. We start with a technical lemma.

Lemma 7 The interval $\langle I, 0\rangle$ can be mapped to $\langle f(I)+m, 0\rangle$ by an $R H P C D$ system with arbitrary constant flows, where $f(x)=a x+b$ is an affine function, $I=(s, t)$ is a 1-dimensional interval and $a, b, m, s, t \in \mathbb{Q}$ are constants.

Proof. We prove this lemma by 3 steps.

Step 1 - Interval $\langle I, 0\rangle$ can be mapped to interval $\langle I, c\rangle$, where $c \in \mathbb{Q}^{+}$, by a bounded PCD with non-comparative guards using flow $(0,1)$. By this, we mean that for any $0 \leq \alpha \leq 1$, point $(s+(t-s) \alpha, 0)$ can be mapped to point $(s+(t-$ $s) \alpha, c$ ) by this bounded PCD. We also use similar terminology throughout.

Step 2 - Suppose we have an affine function $f(x)=a x+b$, and the 1-dimensional rational interval $I=(s, t)$. For any constant $t^{\prime}$ where $t^{\prime} \geq t>s$, define $g=$ $f(t)-f(s)$ and $s^{\prime}=t^{\prime}+|g|$. Assume that $c>|g|+|b|>0$. Then we show the interval $\langle I, c\rangle$ can be mapped to $\left\langle I^{\prime}=\left(t^{\prime}, s^{\prime}\right), 0\right\rangle$ by a bounded PCD system with non-comparative guards; thus meaning that for any $0 \leq \alpha \leq 1$, point $(s+(t-s) \alpha, c)$ can be mapped to point $\left(t^{\prime}+\left(s^{\prime}-t^{\prime}\right) \alpha, 0\right)$, see Fig. 1 . We need to consider 2 cases, $a>0$ and $a<0$.

1. $a>0$. See Fig. 1(a)

(i) Use flow $(1, a)$ to map $\langle(s, t), c\rangle$ to $\langle t,(c, c+|g|)\rangle$;

(ii) Use flow $(1,0)$ to map $\langle t,(c, c+|g|)\rangle$ to $\left\langle t^{\prime},(c, c+|g|)\right\rangle$;

(iii) Use flow $(1,-1)$ to map $\left\langle t^{\prime},(c, c+|g|)\right\rangle$ to $\left\langle\left(t^{\prime}, t^{\prime}+|g|\right), c\right\rangle$;

(iv) Use flow $(0,-1)$ to map $\left.\left.\left\langle\left(t^{\prime}, t^{\prime}+\mid g\right)\right|\right), c\right\rangle$ to $\left\langle\left(t^{\prime}, t^{\prime}+|g|\right), 0\right\rangle$.

2. $a<0$. See Fig. $1(\mathrm{~b})$.

(i) Use flow $(1, a)$ to map $\langle(s, t), c\rangle$ to $\langle t,(c-|g|, c)\rangle$;

(ii) Use flow $(1,0)$ to map $\langle t,(c-|g|, c)\rangle$ to $\left\langle t^{\prime},(c-|g|, c)\right\rangle$;

As we assume $c>|g|+|b|>0$, so $c-|g|>|b|>0$, which means the rectangle $\left\{(x, y)\left|t<x<t^{\prime}, c-\right| g|<y<| g \mid\right\}$ does not intersect with the $x$-axis, hence the following steps make sense.

(iii) Use flow $(1,-1)$ to map $\left\langle t^{\prime},(c-|g|, c)\right\rangle$ to $\left\langle\left(t^{\prime}, t^{\prime}+|g|\right), c-|g|\right\rangle$;

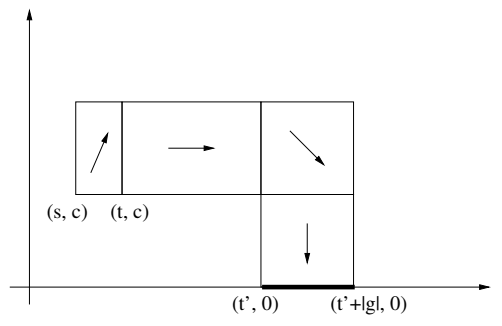

(a) $a>0$

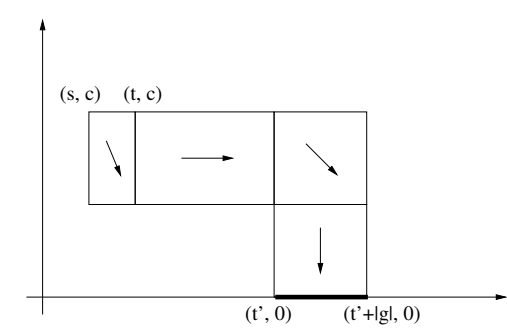

(b) $a<0$

Fig. 1. Lemma 7 Step 2: map $\langle(s, t), c\rangle$ to $\left\langle\left(t^{\prime}, s^{\prime}\right), 0\right\rangle$. 


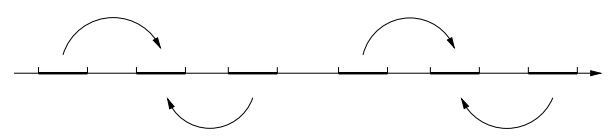

Fig. 2. Idea of Theorem 8: map every two adjacent intervals into one interval

(iv) Use flow $(0,-1)$ to map $\left\langle\left(t^{\prime}, t^{\prime}+|g|\right), c-|g|\right\rangle$ to $\left\langle\left(t^{\prime}, t^{\prime}+|g|\right), 0\right\rangle$.

Note that though some of these steps contains 'triangles', we can define the flows in a rectangular area containing that triangle, thus comparative guards are not required. Note the 'orientation' of the interval is reversed after the mapping.

Step 3 - Using a similar idea we can show the interval $\left\langle I^{\prime}=\left(t^{\prime}, s^{\prime}\right), 0\right\rangle$ can be mapped to $\langle f(I)+m, 0\rangle$, where $f(I)=(f(s), f(t))$ if $a>0$ and $f(I)=$ $(f(t), f(s))$ if $a<0$, by a bounded PCD system with non-comparative guards. We can use only the upper or lower half plane of the PCD. Here we only prove the case when $a>0$ and $f(t)+m<t^{\prime}$ by using the lower half plane, other cases can be proven similarly.

(i) Use flow $(-1,-1)$ to map $\left\langle\left(t^{\prime}, s^{\prime}\right), 0\right\rangle$ to $\left\langle\frac{1}{2}\left(t^{\prime}+f(t)+m\right),\left(-\frac{1}{2} \mid t^{\prime}-f(t)-\right.\right.$ $\left.\left.m|-| g\left|,-\frac{1}{2}\right| t^{\prime}-f(t)-m \mid\right)\right\rangle$

(ii) Use flow $(-1,1)$ to map $\left\langle\frac{1}{2}\left(t^{\prime}+f(t)+m\right),\left(-\frac{1}{2}\left|t^{\prime}-f(t)-m\right|-|g|,-\frac{1}{2} \mid t^{\prime}-\right.\right.$ $f(t)-m \mid)\rangle$ to $\langle(f(s)+m, f(t)+m), 0\rangle$.

Combining Step 1,2 and 3 we get the result of the lemma. We need a 2location RHPCD system with arbitrary constant flows and hence each location is a bounded PCD system with non-comparative guards. In location 1 we realize Step 1 and jump to location 2, i.e., the guards are $\left\langle s_{i} \leq x<t_{i}, y=c\right\rangle$. In location 2 we realize Step 2 and Step 3 together because Step 2 only uses the upper plane of a PCD and Step 3 only requires the lower plane of a PCD.

Theorem 8 A 1-PAM with $n$ intervals can be simulated by an RHPCD with $\left\lceil\log _{2} n\right\rceil+3$ locations such that one of the variables has arbitrary constant flows.

Proof. Suppose PAM $\mathcal{A}$ is defined by $f(x)=a_{i} x+b_{i}$ if $x \in I_{i}$, with $1 \leq i \leq n$ and $I_{i}$ are rational intervals. Let the left and right endpoints of $I_{i}$ be $s_{i}$ and $t_{i}$ respectively. First we show that this PAM can be simulated straightforwardly by an $n+1$-location RHPCD with arbitrary constant flows. We need a single location $p$ as the global state and $n$ locations $q_{i}$ for each interval $I_{i}, 1 \leq i \leq n$.

1. In location $p$, we define the corresponding points of the PAM $\mathcal{A}$ on interval $\left\langle\left(s_{1}, t_{n}\right), 0\right\rangle$. We then map each $\left\langle I_{i}, 0\right\rangle$ to the interval $\left\langle I_{i}, c\right\rangle$, where $c=\left|\max \left\{\left|a_{i}\right|\right\}\left(t_{n}-s_{1}\right)\right|+\max \left\{\left|b_{i}\right|\right\}$. (See Lemma 7, Step 1). The transition guards of $p$ are: $\left\langle s_{i} \leq x<t_{i}, y=c\right\rangle$, in which we jump to $q_{i}$.

2. In location $q_{i}$, map $\left\langle I_{i}, c\right\rangle$ to $\left\langle f\left(I_{i}\right), 0\right\rangle$ (see Lemma 7, Step 2\&3). The transition guard of $q_{i}$ is : $\left\langle s_{1} \leq x<t_{n}, y=0\right\rangle$, with a jump to location $p$. 
The above method requires $n+1$ locations for a PAM with $n$ intervals. We now give an improved method using an RHPCD with only $\left\lceil\log _{2} n\right\rceil+3$ locations.

Suppose the PAM $\mathcal{A}$ contains $n$ intervals. For every $n \neq 2^{d}, d \in \mathbb{N}$, there exists a minimum integer $k \in \mathbb{N}$ such that $\log _{2}(n+k)=\left\lceil\log _{2} n\right\rceil$. The PAM $\mathcal{A}$ can be expanded to $\mathcal{A}^{\prime}$ such that $f(x)=a_{i} x+b_{i}$ if $x \in I_{i}$, where $i \in\{1, \ldots, n\}$. For every $i^{\prime} \in\{1, \ldots, k\}$, the length of each new added interval is given by $\left|I_{\varepsilon_{i^{\prime}}}\right|=\epsilon$, and the corresponding affine function is $f(x)=x$. This expansion does not change the dynamics of the PAM $\mathcal{A}$, thus we assume $n=2^{d}, d \in \mathbb{Z}$.

Again, let the left endpoint and the right endpoint of $I_{i}$ be $s_{i}$ and $t_{i}$ respectively. Define $c$ to be $c=\left|\max \left\{\left|a_{i}\right|\right\}\left(t_{n}-s_{1}\right)\right|+\max \left\{\left|b_{i}\right|\right\}$ and $l$ to be $l=\left|t_{n}-s_{1}\right|$.

Step 1 Define the PAM on interval $\left\langle\left(s_{1}, t_{n}\right), 0\right\rangle$. For every $i \in\{1,2, \ldots, n\}$, map $\left\langle I_{i}, 0\right\rangle$ to interval $\left\langle I_{i}, 2(n-i+1) c\right\rangle$. (See Lemma 7, Step 1). In this step each interval is mapped to a different height $y=2(n-i+1) c$. There is a $2 c$-length 'gap' between every two intervals $I_{i}$ and $I_{i+1}$ and $I_{i}$ is higher than $I_{i+1}$. In Lemma 7 Step 2 this clearly prevents intersections in the following step.

Step 2 Map each interval $\left\langle I_{i}, 2(n-i+1) c\right\rangle$ to $\left\langle\left(f\left(I_{i}\right)+2(n-i+1) l, 0\right\rangle\right.$. (See Lemma 7 , Step 2). Then between every two intervals there is a 'gap' whose length is $l$.

Step 3 For $i$ from 1 to $\frac{n}{2}$, let $j=2 i-1$, we can find an undefined interval between $\left\langle f\left(I_{j}\right)+2(n-j+1) l, 0\right\rangle$ and $\left\langle f\left(I_{j+1}\right)+2(n-j+2) l, 0\right\rangle$ of length l. By the proof of Lemma 7 (Step 3), we can map $\left\langle f\left(I_{j}\right)+2(n-j+1) l, 0\right\rangle$ using the upper plane and $\left\langle f\left(I_{j+1}\right)+2(n-j+2) l, 0\right\rangle$ using the lower plane to this interval.

Step 4 Repeat Step 2 for $\log _{2}(n)$ times until only 1 interval, $I_{f}$, remains.

Step 5 If the orientation of $I_{f}$ is 'reversed' with respect to the initial interval of the PAM $\mathcal{A}$, then map $I_{f}$ to this initial interval; otherwise, we reverse it before mapping it to the initial interval.

Step 1, 2 and 5 each require 1 location. Step 3 and Step 4 require $\log _{2} n$ locations, thus $\left(\log _{2} n\right)+3$ locations are required.

The difficulty of simulating a 1-PAM by a 2-PCD is that regions cannot overlap in a PCD, i.e., one region has only one deterministic constant flow. Thus it is impossible to map several different intervals into a single interval under a 2-PCD, leading us to believe that $\Omega\left(\log _{2} n\right)$ is a lower bound of the number of locations required to simulate an $n$-interval 1-PAM by an RHPCD with arbitrary constant flows.

Example 9 We give an example of a 1-PAM below and show how to simulate it by a RHPCD with arbitrary constant flows in Figs. 3, 4.

$$
f(x)=\left\{\begin{array}{lll}
2 x, & \text { if } & x \in[0,1) \\
-x+2, & \text { if } \quad x \in[1,2]
\end{array}\right.
$$

Let the initial point be $x_{0}$. The initial location of the HPCD is $A-1$, with variables $(x, y)=\left(x_{0}, 0\right) . P C D$ A-1 corresponds to Theorem 8, Step 1. PCD A-2 separates 


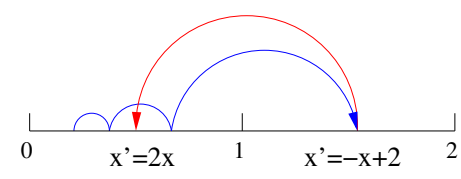

(a) A 1-dim. Piecewise Affine Map

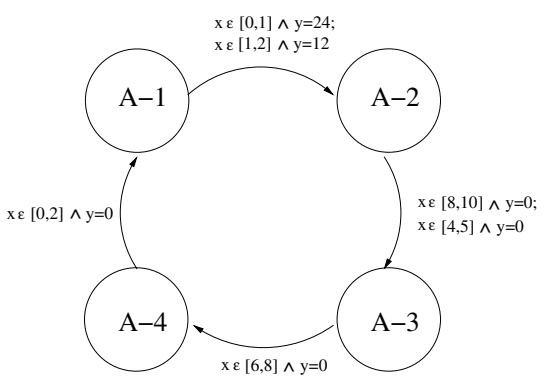

(b) RHPCD with arbitrary constant flows

Fig. 3. The 1-PAM with its equivalent HPCD

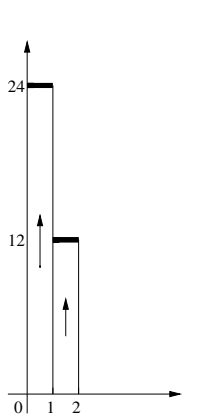

(a) A-1

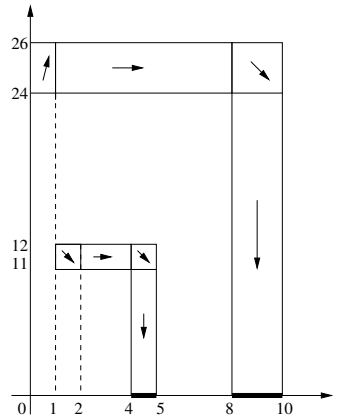

(b) A-2

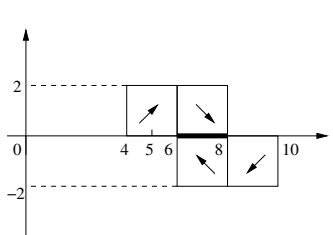

(c) A-3

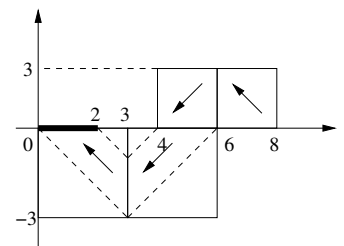

(d) A-4

Fig. 4. The 2-PCDs of the HPCD in Fig 3b (transition guards in bold).

each interval onto the $x$ axis (Theorem 8, Step 2). PCD A-3 combines together these two intervals (Theorem 8, Step 3). Finally, in A-4, as the final interval $[6,8]$ has the same orientation as the initial interval $[0,2]$, we reverse it before mapping it back to the initial interval (Theorem 8, Step 5).

We now show that an RHPCD with linear resets can simulate a 1-PAM.

Lemma 10 The interval $\langle I, 0\rangle$ can be mapped to $\langle f(I)+m, 0\rangle$ by an $R H P C D$ system with linear resets, where $f(x)=a x+b$ is an affine function, $I=(s, t)$ is a 1-dimensional interval and $a, b, m, s, t \in \mathbb{Q}$ are constants.

Proof. The proof is similar to the proof of Lemma 7. We still prove by 3 steps.

Step 1 First map the interval $\langle I, 0\rangle$ to the interval $\langle I, c\rangle$ by flow $(0,1)$. Define the transition guard to be $\langle I, c\rangle$, which jumps to location 2 with linear reset: $x^{\prime}=|a| x, y^{\prime}=y$.

Step 2 Using the similar idea in Lemma 7 Step 2, we can map the interval $\langle|a| I, c\rangle$ to the interval $\left\langle\left(t^{\prime}, t^{\prime}+|g|\right), 0\right\rangle$ by the flows $(1,1)$ if $(a>0)$ or 


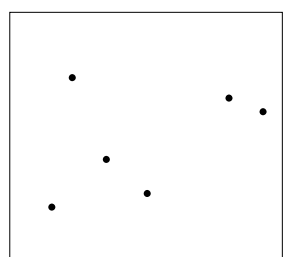

(a) Set of Points $V \subseteq \mathbb{R}^{2}$

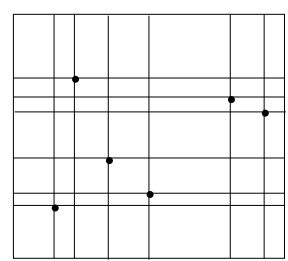

(b) Its Rectilinear Tessellation

Fig. 5. Rectilinear Tessellation of the plane.

$(1,-1)$ if $a<0,(1,0),(1,-1)$ and $(0,-1)$, where $t^{\prime}$ and $g$ are the same defined as in Lemma 7.

Step 3 Exactly the same as Lemma 7 Step 3.

Theorem 11 A 1-PAM with $n$ intervals can be simulated by an RHPCD containing $\left\lceil\log _{2} n\right\rceil+3$ locations with linear resets.

Proof. Apply Lemma 10 instead of Lemma 7 in the proof of Theorem 8.

Corollary 12 An RHPCD with linear resets is equivalent to an RHPCD with affine resets.

Proof. Immediately from the results of [3] and Theorem 11.

Definition 13 (Rectilinear Tessellation) - Let $V \subseteq \mathbb{R}^{2}$ be a finite set of 2-dimensional points. We define a rectilinear tessellation of the plane as a tessellation by rectangles by identifying with each $v \in V$ a splitting of the plane into four quadrants, parallel to the $x$ and $y$ axes. See Fig. 5(a) and Fig. 5(b).

Lemma 14 Let $\mathcal{H}$ be an $R H P C D$. There exists an $R H P C D, \mathcal{H}_{R}$, which is topologically equivalent to $\mathcal{H}$, such that $\mathcal{H}_{R}$ has an injective edge-to-edge successor function which preserves local coordinates.

Proof. Given the $n$-location RHPCD $\mathcal{H}$, we define the rectilinear tessellation of $\mathcal{H}$ in the following way. Let $V_{i} \subseteq \mathbb{Q}^{2}$ be the set of points defining the PCD regions of location $l_{i}$ of $\mathcal{H}$ and let $V=\cup V_{i}$ for $1 \leq i \leq n$. We assume without loss of generality that $V \subseteq \mathbb{Z}^{2}$ by forming a (topologically) equivalent HPCD with all points multiplied by the least common multiple of the denominators of coordinates of points in $V$. We form a rectilinear tessellation of the plane by set of points $V$. Note that each location of $\mathcal{H}$ can thus be defined on the same set of regions, but allowing a region in different locations to have a different derivative.

We define derivatives of the form $\{( \pm 1,0),(0, \pm 1)\}$ as straight flows and derivatives of the form $\{( \pm 1, \pm 1),( \pm 1, \mp 1)\}$ as diagonal flows. Our next step is to further decompose the rectilinear tessellation of the plane such that any non-square region containing a diagonal flow in any location is split into a finite 
number of square regions, each of which contains the same diagonal flow. To perform this step, let $R$ by any (non-square) rectangle such that there exists a location $l_{i}$ where the derivative of $R$ in $l_{i}$ is a diagonal flow. Let $\left(r_{x}, r_{y}\right) \in \mathbb{Z}^{2}$ be the bottom left point of $R$ and $\left(r_{x}^{\prime}, r_{y}^{\prime}\right) \in \mathbb{Z}^{2}$ be its upper right point. Then we add all points $\left\{(i, j) \mid r_{x} \leq i \leq r_{x}^{\prime}, r_{y} \leq j \leq r_{y}^{\prime}\right\} \subseteq \mathbb{Z}^{2}$ to set $V$ and recompute the rectilinear tessellation of $\mathcal{H}$. There are a finite number of bounded regions in $\mathcal{H}$ and thus this procedure eventually halts giving a final set of points $V^{\prime}$ defining the regions shared by all locations of the new RHPCD $\mathcal{H}_{R}$.

In each location of $\mathcal{H}_{R}$, only square regions have diagonal flows, thus each edge is mapped to exactly one other under $\mathcal{H}_{R}$. Clearly then, local coordinates of each edge are preserved by the edge-to-edge successor function by rectilinearity of the plane partition and since each region has elementary flows.

Theorem 15 The reachability problem is decidable for an RHPCD.

Proof. Given $\mathcal{H}$, we can apply the rectilinear tessellation technique of Lemma 14 to form an RHPCD $\mathcal{H}_{R}$ satisfying the conditions of the lemma. Let $\left(l_{0}, \alpha\right)$ be the initial state of the system and $\left(l_{f}, \beta\right)$ be the final point of the system, where $\alpha, \beta \in \mathbb{Q}^{2}$. In the same way as in Lemma 14 , these points can be transformed so that $\alpha, \beta \in \mathbb{Z}^{2}$ are the initial and final points under $\mathcal{H}_{R}$.

Since the edge-to-edge successor function of $\mathcal{H}_{R}$ is injective, we can form a finite graph with vertices labelled $\left(e_{i}, k\right)$ where $\left(e_{i}, k\right)$ is an edge in location $l_{i}$ of $\mathcal{H}_{R}$. Each vertex is connected to exactly one other, according to the injective edge-to-edge successor function. Since local coordinates of points on edges are preserved by this function by Lemma 14, reachability becomes trivial since the local coordinate of $y$ must be the same as $x$ and we can simply traverse the graph until we reach the correct edge in some location or else detect a cycle.

Definition 16 (1-POM) Let $f$ be a 1-PAM. We call $f$ a one-dimensional piecewise offset map (1-POM) if $f(x)=x+b_{i}$ for all $x \in I_{i}$.

Corollary 17 A 1-POM can be simulated by an RHPCD, and an RHPCD an be simulated by a 1-POM.

The following theorem shows a relationship between the additional computational powers of affine resets and arbitrary constant flows.

Theorem 18 A $k$-location RHPCD with arbitrary constant flows can be simulated by a $k$-location $R H P C D$ with affine resets for any $k \geq 1$.

Finally, we introduce nondeterminism to the RHPCD model.

Theorem 19 The reachability problem for a nondeterministic RHPCD system is NP-hard.

Proof. We use a reduction of the Subset Sum Problem (SSP): given a finite set of positive integers $A$ and a positive integer $N>0$, is there a subset $A^{\prime} \subseteq A$ such 


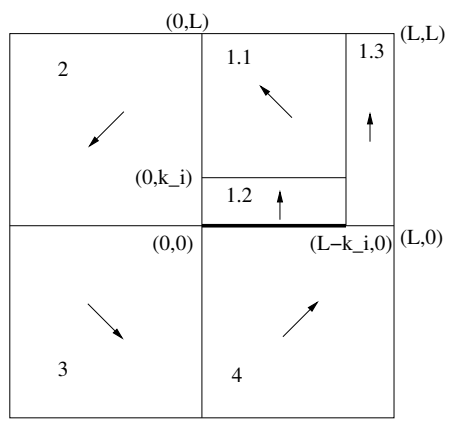

Fig. 6. SSP simulated by a nondeterministic RHPCD with arbitrary constant flows. The bold line denotes the transition guard.

that the sum of the elements in $A^{\prime}$ is exactly $N$ ? i.e., if $A=\left\{k_{1}, k_{2}, \ldots, k_{n}\right\} \subseteq \mathbb{Z}^{+}$, is there a subset $A^{\prime}=\left\{k_{t_{1}}, k_{t_{2}}, \ldots, k_{t_{m}}\right\} \subseteq A$ such that $\sum_{j=1}^{m} k_{t_{j}}=N$ ?

To simulate an $n$ element instance of SSP, we define an $(n+2)$-location nondeterministic RHPCD. Divide each location $i(1 \leq i \leq n)$ into four squares labelled 1 to 4 ; see Fig. 6 . Each square has length $L$, where $L>N+\max \left\{\left|k_{i}\right|\right\}+\varepsilon$, $\varepsilon \in(0,1)$. Define the left lower corner point of square 1 to be the original point $(0,0)$ and the right upper corner point of square 1 to be the point $(L, L)$ and the other regions are located as in Fig. 6 . For regions $2,3,4$ we need elementary flows $(-1,-1),(1,-1)$ and $(1,1)$, respectively. For each location $i(1 \leq i \leq n)$, divide square 1 into three subregions $1.1,1.2$ and 1.3 by internal guards $\left\langle\left(0, L-k_{i}\right), k_{i}\right\rangle$ and $\left\langle L-k_{i},(0, L)\right\rangle$. Then the region 1.1 is defined as a square of length $L-k_{i}$ and regions 1.2 and 1.3 are two rectangles located inside square 1 as in Fig. 6. The flows of region 1.1, 1.2 and 1.3 are defined as $(-1,1),(0,1)$ and $(0,1)$, respectively. Because $L>N+\max \left\{\left|k_{i}\right|\right\}+\varepsilon$, the point $(0, N+\varepsilon)$ is possible to reach from the $x$-axis in each location $i$. Define the transition guard of each location $i$ to be $\langle 0<x<L, y=0\rangle$, which jumps to any one of the locations from location $i+1$ to $n+1$. At last let location 0 be the starting location from which we can jump to location 1 to $n$, and location $n+1$ be the final location where all the trajectories move with flow $(1,0)$. Clearly, the SSP instance has a solution if the point $(0, N+\varepsilon)$ in any location $i(1 \leq i \leq n)$ can be reached from $(\varepsilon, 0)$ in location 0 . Note that the construction of the corresponding RHPCD can be done in time polynomial in the SSP instance size.

\section{Conclusion}

We showed decidability of reachability for Restricted Hierarchical Piecewise Constant Derivative (RHPCD) systems. The complexity of this problem is interesting but is currently unresolved. We then showed that adding: comparative guards, arbitrary constant flows or linear resets to an RHPCD makes the problem equivalent to reachability for 1-PAMs, which is a long standing open problem. We also showed that adding nondeterminism to RHPCDs leads to NP-hardness of reachability. In this paper we focused on reachability problems, but stability, 
convergence and control problems for low-dimensional linear hybrid systems are also important topics under active research.

Acknowledgements: We would like to thank the anonymous referees for their very useful suggestions and comments.

\section{References}

1. R. Alur, C. Courcoubetis, N. Halbwachs, T. A. Henzinger, P. H. Ho, X. Nicollin, A. Olivero, J. Sifakis, and S. Yovine. The algorithmic analysis of hybrid systems. Theoretical Computer Science, 138(1):3-34, 1995.

2. E. Asarin, O. Maler, and A. Pneuli. Reachability analysis of dynamical systems having piecewise constant derivatives. Theoretical Computer Science, 138:35-65, 1995.

3. E. Asarin, V. Mysore, A. Pneuli, and G. Schneider. Low dimensional hybrid systems - decidable, undecidable, don't know. Information and Computation, 211:138$159,2012$.

4. E. Asarin and G. Schneider. Widening the boundary between decidable and undecidable hybrid systems. In CONCUR'02, volume 2421 of $L N C S$, pages 193-208. Springer, 2002.

5. E. Asarin, G. Schneider, and S. Yovine. Algorithmic analysis of polygonal hybrid systems, part I: Reachability. Theoretical Computer Science, 379:231-265, 2007.

6. A. Balluchi, L. Benvenuti, M. di Benedetto, C. Pinello, and A. SangiovanniVincentelli. Automotive engine control and hybrid systems: challenges and opportunities. Proceedings of the IEEE, 88(7):888-912, 2000.

7. N. Bauer, S. Kowalewski, and G. Sand. A case study: Multi product batch plant for the demonstration of control and scheduling problems. In ADPM, pages 969-974, Dortmund, Germany, 2000.

8. V. Blondel and J. Tsitsiklis. When is a pair of matrices mortal? Information Processing Letters, 63:283-286, 1997.

9. V. Blondel and J. N. Tsitsiklis. A survey of computational complexity results in systems and control. Automatica, 36:1249-1274, 2000

10. T. Henzinger and J.-F. Raskin. Robust undecidability of timed and hybrid systems. In Hybrid Systems: Computation and Control, volume 1790, pages 145-159, 2000.

11. O. Kurganskyy, I. Potapov, and F. Sancho-Caparrini. Reachability problems in low-dimensional iterative maps. International Journal of Foundations of Computer Science, 19(4):935-951, 2008.

12. O. Maler and A. Pnueli. Reachability analysis of planar multi-linear systems. In CAV93, volume LNCS 697, pages 194-209. Springer-Verlag, 1993.

13. P. Prabhakar, V. Vladimerou, M. Viswanathan, and G. Dullerud. A decidable class of planar linear hybrid systems. In Hybrid Systems: Computation and Control, volume 4981, pages 401-414, 2008. 